\title{
Experimental study of hydrodynamics in PTFE-grafts
}

\author{
I. Van Tricht ${ }^{1}$, D. De Wachter ${ }^{1}$, J.H.M Tordoir ${ }^{2}$ and P. Verdonck ${ }^{1}$ \\ ${ }^{\prime}$ Hydraulics Laboratory, Institute Biomedical Technology, Ghent \\ University (RUG), Ghent, Belgium. \\ ${ }^{2}$ Department of Surgery, Academic Hospital Maastricht (AHM), \\ Maastricht, The Netherlands.
}

\begin{abstract}
An experimental model has been built to investigate the hydrodynamics in vascular access grafts for hemodialysis. Two types polytetrafluorethyleen (PTFE)-grafts are tested: a straight and a tapered graft. For both grafts, two scenarios are studied. The first is the "normal" scenario: mean inlet pressure is kept at $100 \mathrm{~mm} \mathrm{Hg}$. In the second, "low resistance", mean outlet pressure is held at $20 \mathrm{~mm} \mathrm{Hg}$. Flow rate varies between 500 and $1500 \mathrm{ml} / \mathrm{min}$. Temporal pressure curves and maximum velocity curves are measured on different locations in the model. Spatial pressure profiles for mean and pulse pressure are derived out of these measurements. Pulse pressure is higher in the tapered graft than in the straight and increases towards the arterial anastomosis of the tapered graft at high flow rates. Flow in the vein has become pulsatile where flow in normal peripheral veins is constant.
\end{abstract}

\section{Introduction}

Patients with renal failure need a renal replacement therapy. One of the possible therapies is hemodialysis: the excess of water is drained and blood is purified outside the human body in an artificial kidney. In this artificial kidney, blood and dialysis fluid are circulated countercurrently on opposite sides of a porous membrane which allows the passage of waste metabolites and water but limits the transfer of blood cells and proteins. To obtain a sufficiently high blood flow $(400 \mathrm{ml} / \mathrm{min})$ through the extracorporal circulation in the artificial kidney, a vascular access is needed [1]. Atero venous fistula (AVF) and PTFE-grafts are commonly used to 


\section{4}

Simulations in Biomedicine $V$

create a vascular access. In the case of unsuitable arteries and/or veins to create an AVF, like in diabetics and elderly, a PTFE-graft is used as a vascular access [2]. In this article we will focus on PTFE-grafts. Normal flow rate is for a well functioning graft $1000 \mathrm{ml} / \mathrm{min}$, which is much higher than the 400 needed in the extracorporal system. The major problems of PTFE-grafts are venous anastomosis stenoses, venous stenoses and thromboses at low flow rates $(<600 \mathrm{ml} / \mathrm{min})$ [7]. Because these phenomena are influenced by flow conditions, the complex hydrodynamics are investigated using an in vitro model.

\section{Methods}

\subsection{Vascular access model}

A vascular access model is built with silicon arteries and veins and a polytetrafluorethyleen (PTFE) -graft.

Silicon arteries and veins are constructed such that the properties: diameter, relative distensibility (2) and compliance (1), are similar as in patients [3] [4]. The diameter (D) of the arteries is $\pm 4 \mathrm{~mm}$ and of veins is $\pm 6 \mathrm{~mm}$.

$$
\begin{aligned}
\text { Compliance } & =\frac{\delta V}{\delta p} \\
\text { Relative Distensibility } & =\frac{D_{\text {systole }}-D_{\text {diastole }}}{D_{\text {systole }}}
\end{aligned}
$$

Vessel compliance, is a parameter which determines the buffer capacity of a blood vessel: the higher the compliance, the more a blood vessel can store blood during systole to release it during diastole. $\delta V$ is an elementary volume change and $\delta p$ is an elementary pressure change during the cardiac cycle. The relative distensibility, derived from the compliance, expresses the diameter changes during a simulated cardiac cycle. $D_{\text {systole }}$ is the diameter at systolic pressure; $D_{\text {diastole }}$ is the diameter at diastolic pressure. In our model, the relative distensibility is measured using the wall track method (Pie Medical - Maastricht - The Netherlands) and are found to be $4 \%$, like in patients.

Two types of PTFE-grafts are studied: a straight and a tapered one. The straight graft has a constant diameter of $6 \mathrm{~mm}$ and a total length of $34 \mathrm{~cm}$. The diameter of the tapered graft is $4 \mathrm{~mm}$ at the arterial inlet and increases smoothly up to $7 \mathrm{~mm}$ the next $50 \mathrm{~mm}$, the total length is $34 \mathrm{~mm}$.

The PTFE-graft is sewed to a silicon artery and vein by a vascular surgeon, see figure 1.

\subsection{The setup}

Blood simulating fluid is used in the setup. It is a mixture of $40 \%$ glycerine and $60 \%$ water with a dynamic viscosity $(\mu)$ of $3,75 \mathrm{mPa}$.s and a density $(\rho)$ of 1102,8 $\mathrm{kg} / \mathrm{m}^{3}$ at $25^{\circ} \mathrm{C}$. 


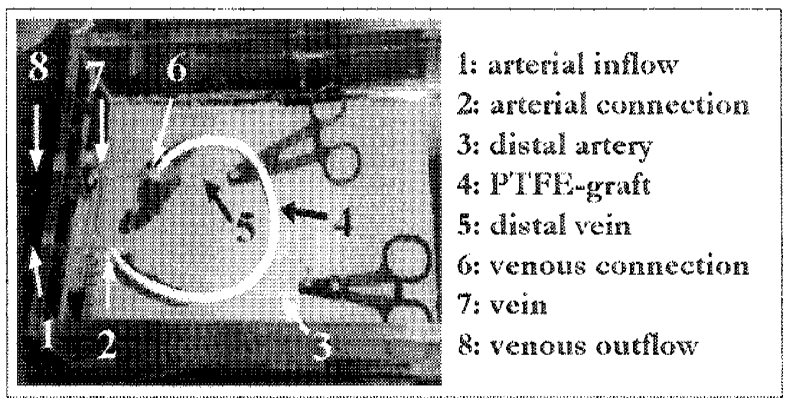

Figure 1: The vascular access model

A pulsatile pump (P) (Harvard apparatus, pulsatile pump 1421, Model 55-3339) pumps the fluid out of a reservoir (R) into a windkessel (W). The fluid flows from the windkessel into the vascular access model (VA-M) which is drained into the reservoir again, see figure 2. Physiologic pressure and flow waves are generated at the arterial inlet of the vascular access model. The maximum pressure in the cycle is systolic pressure, the minimum pressure is diastolic pressure and the difference between both is pulse pressure, see figure 3 . Mean pressure is the time-averaged pressure of a cycle. Heart rate is set 90 beats per minute (BPM); a full cycle takes 0,667 seconds.

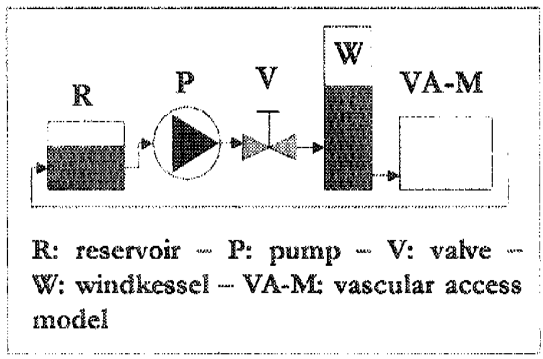

Figure 2: The setup

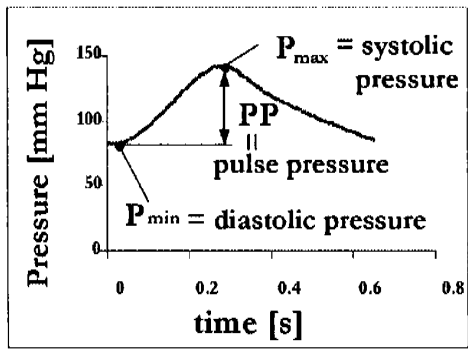

Figure 3: Generated pressure wave

Two scenarios are considered in the setup. Mean arterial inlet pressure is kept constant at $100 \mathrm{~mm} \mathrm{Hg}$ in the "normal" scenario. Mean venous outlet pressure is held to $20 \mathrm{~mm} \mathrm{Hg}$ in the "low resistance" scenario. Pressure is measured with a fluid filled catheter connected to a pressure transducer (BD).

Flow rates are varied between $500 \mathrm{ml} / \mathrm{min}$ and $1500 \mathrm{ml} / \mathrm{min}$ in each scenario. A volume flow of $500 \mathrm{ml} / \mathrm{min}$ corresponds with thrombosis risk, $1000 \mathrm{ml} / \mathrm{min}$ with a normal functioning access graft and $1500 \mathrm{ml} / \mathrm{min}$ with hemolysis risk. Mean flow is measured volumetrically. The velocity $(v)$ change in time is measured in the center of the blood vessel with an ultrasound machine (VINGMED CFM 800, GE Medical systems Benelux). 
Considering all these parameters, it can be found that the Womersley number (3), varies between 6,25 and 9,40 and the highest Reynolds number (4), is 2800 . D is the diameter of the blood vessel, $\omega$ is the pulsatility of the fluid, $\rho$ is the density of the fluid, $\mu$ is the dynamic viscosity and $v$ is the mean velocity.

$$
\begin{aligned}
\alpha & =\frac{D}{2} \sqrt{\frac{\omega \rho}{\mu}} \\
R e & =\rho \frac{v D}{\mu}
\end{aligned}
$$

\section{Results}

Pressure variation in time is measured at different locations in the vascular access model: at the arterial inlet (" $0 \mathrm{~cm}$ "), $2 \mathrm{~cm}$ before the arterial anastomosis("13 cm"), in the arterial anastomosis("15 cm"), different points in the graft, in the venous anastomosis("49 cm"), $3 \mathrm{~cm}$ after the venous anastomosis ("52 cm") and at the venous outflow ("66 cm"). Out of these temporal pressure curves, spatial pressure curves can be calculated for mean and pulse pressure. In figure 4 spatial curves for mean and pulse pressure are plotted in the "normal" scenario at a flow rate of 1000 $\mathrm{ml} / \mathrm{min}$. In this figure difference between both graft types can be seen.

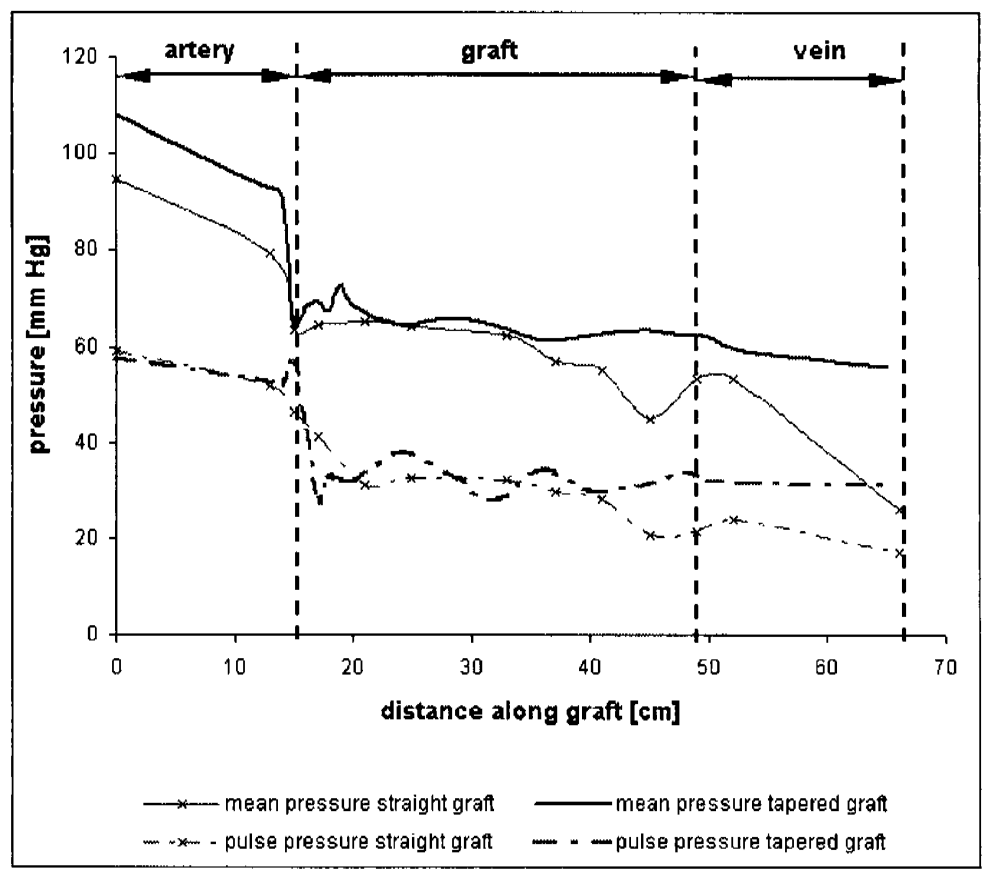

Figure 4: Comparing "straight" and "tapered" graft types at $1000 \mathrm{ml} / \mathrm{min}$ 


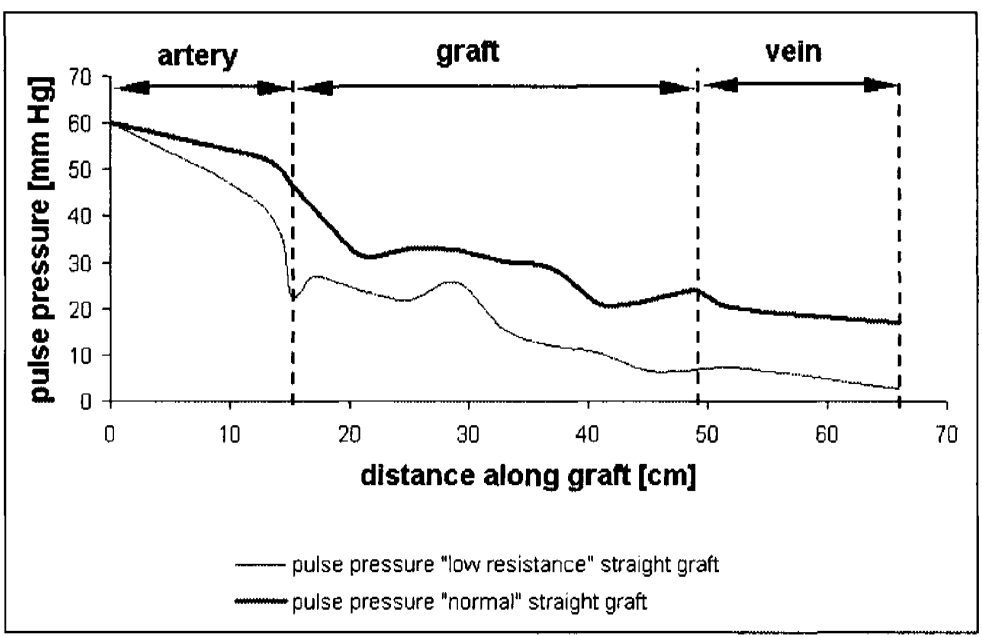

Figure 5: Comparing "normal" and "low resistance" scenario at $1000 \mathrm{ml} / \mathrm{min}$ in the straight graft

In figure 5 pulse pressure in the different scenarios are plotted for a flow rate of $1000 \mathrm{ml} / \mathrm{min}$ in the straight graft.

In figures $6,7,8$ and 9 time varying velocity magnitude measured in the center of the blood vessels in the straight graft model in the "normal" scenario are shown.

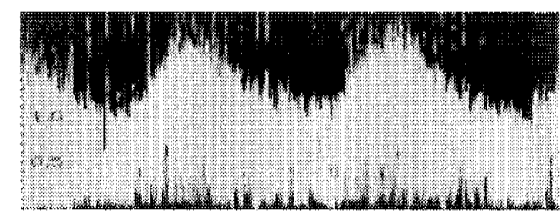

Figure 6: Velocity measured at $15 \mathrm{~cm}$, $1000 \mathrm{ml} / \mathrm{min}$

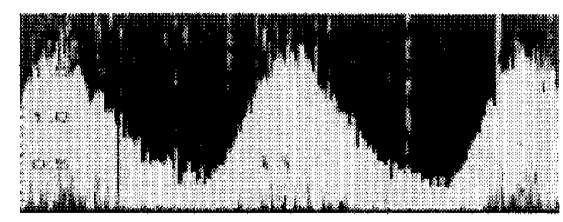

Figure 8: Velocity measured at $15 \mathrm{~cm}$, $500 \mathrm{ml} / \mathrm{min}$

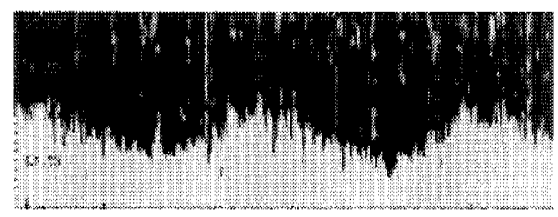

Figure 7: Velocity measured at $49 \mathrm{~cm}$, $1000 \mathrm{ml} / \mathrm{min}$

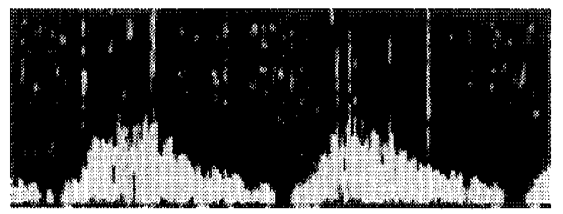

Figure 9: Velocity measured at $49 \mathrm{~cm}$, $500 \mathrm{ml} / \mathrm{min}$ 


\section{Discussion and Conclusion}

As pulse pressure is higher in the tapered graft than in the straight graft (see Figure 4), the artery and vein get an increased function as compliance chambers in the tapered graft. For the tapered graft another observation is that pulse pressure increases at the arterial anastomosis, especially at high flow rates, which means that the artery at the arterial anastomosis acts as a buffer chamber. Fluid is collected in that artery during systole and drained during diastole. This might induce wall fatigue around the arterial anastomosis resulting in intimal hyperplasia leading to stenosis.

Pressure loss at the arterial anastomosis of the tapered graft is twice as much as in the straight graft. Pressure in the vein is higher than the physiological $20 \mathrm{~mm} \mathrm{Hg}$ in the tapered graft model.

In both grafts, pressure recovery exists at the inflow of the graft, however the phenomenon is stronger and over a smaller distance in the tapered graft. At the venous anastomoses, were the diameter mismatch is lower, also a lower local pressure loss is seen.

Comparing the "normal" and the "low resistance" pressure data, a difference in the pulse pressures can be seen. Pulse pressure is higher in the "normal" case, thus artery and vein act more as a buffer chamber in that case (see Figure 5).

Mean velocity increases at the anastomoses, which may confirm a local effective diameter decrease and consequently the existence of vortexes [5][6].

The flow through the model is a typical low-resistance flow [7] with a high maximum $v_{m a x}$ and mean velocity and a positive minimum velocity $v_{\min }$. This can be seen in the velocity images captured with pulsed Doppler (see figures 6,7,8,9). At low flow rates $(500 \mathrm{ml} / \mathrm{min}) \Delta v\left(=v_{\max }-v_{\min }\right)$ increases and the mean velocity is lower. The minimum velocity decreases down to $0.3 \mathrm{~m} / \mathrm{s}$ in the center of the blood vessel. Near the vessel wall, velocities will be lower or even zero. This has an effect on shear stress (5), which is proportional to the radial velocity gradient in the blood vessel $\left(\frac{\delta v}{\delta r}\right)$.

$$
\tau=\mu \frac{\delta v}{\delta r}
$$

Near the vessel wall very low shear stresses and shear stress gradients appear, which can induce the development of intimal hyperplasia and stenoses [7]. Another observation is that the flow in the vein is still pulsatile, $\Delta v$ is $\pm 0,4 \mathrm{~m} / \mathrm{s}$, where in normal peripheral veins flow is quasi constant. This venous pulsation combined with the high pressure level, might also induce vessel wall fatigue. 


\section{Acknowledgments}

The research of Ilse Van Tricht is supported by the Institute for the Promotion of Innovation by Science and Technology in Flanders (IWT).

Dirk De Wachter is a post-doctoral Fellow of the Fund for Scientific Research Flanders, Belgium.

The authors would like to acknowledge the suggestions of the colleagues of the Hydraulics Laboratory Nikos Stergiopulos for the use of his pulsatile pump.

\section{References}

[1] Uldall, R., Replacement of Renal Function by Dialysis, Kluwer Academic Publishers, chapter 10A, pp. 277-292, 1996.

[2] Butler, C. \& Tilney, N., Replacement of Renal Function by Dialysis, Kluwer Academic Publishers, chapter 10B, pp. 292-304, 1996.

[3] Kosch, M., Acute effects of haemodialysis on endothelial function and large artery elasticity. Nephrol Dial Transplant, 16, pp. 1663-1668, 2001.

[4] Hofstra, L., Mismatch in elastic properties around anastomoses of interposition grafts for hemodialysis access. J Am Soc Nephrol, 5(5), pp. 1243-1250, 1994.

[5] Ohja, Influence of angle on wall shear stress distribution for an end-to-side anastomosis. J Vasc Surg, 19(6), pp. 646-655, 1994.

[6] Xue-Mei, Hemodynamic factor at the distal end-to-sde anastomosis of a bypass graft with different pos:dos flow ratios. J Biomech Eng, 123(3), pp. $270-6,2001$.

[7] European basic multidisciplinary hemodialysis access course. Blood Purification, 20(4), pp. 409-440, 2002.

[8] Verdonck, P., Intra and Extracorporeal Cardiovascular Fluid Dynamics. WIT Press: University of Ghent, Belgium, 1998. 
wy Transactions on Biomedicine and Health vol 6, (c) 2003 WIT Press, www.witpress.com, ISSN 1743-3525 